\title{
Plasma Free Amino Acid and Carnitine Levels in Pregnant Women with Hydatidiform Mole: A Case-Controlled Study
}

\author{
Hacer Uyanıkoğlu ${ }^{1}$, Ismail Koyuncu ${ }^{2}$, Sibel sak $^{3}$, adnan incebiyik ${ }^{3}$, nese gul hilali ${ }^{3}$, emin \\ tasduzen $^{3}$, ataman gonel ${ }^{1}$, and Muhammet Erdal $\mathrm{SAK}^{3}$ \\ ${ }^{1}$ Harran University Faculty of Medicine \\ ${ }^{2}$ Harran University \\ ${ }^{3}$ Affiliation not available
}

June 10, 2021

\begin{abstract}
Objective: To compare plasma free amino acid (FAA) and carnitine levels in pregnant women with and without hydatidiform mole (HM). Methods: This prospective study was conducted in patients admitted to Harran University Medical Faculty Hospital Obstetrics Clinic between January and November 2018. Twenty-three pregnant women with HM, and 24 healthy pregnant women as controls were enrolled in the study. The venous blood samples were collected, and, FAA and carnitine concentrations were measured in plasma using liquid chromatography/tandem mass spectrometry (LC-MS/MS). Results: The levels of alanine, arginine, and valine from the 14 amino acids examined were significantly lower in the HM group than in the healthy group $(475.46 \pm 103.55 \mu \mathrm{mol} / \mathrm{L}$ vs $556.99 \pm 105.16 \mu \mathrm{mol} / \mathrm{L}, \mathrm{p}=0.019 ; 428.87 \pm 138.91 \mu \mathrm{mol} / \mathrm{L}$ vs $547.11 \pm 140.20 \mu \mathrm{mol} / \mathrm{L}, \mathrm{p}=$ 0.009 ; and $290.96 \pm 97.10 \mu \mathrm{mol} / \mathrm{L}$ vs $358.61 \pm 75.40 \mu \mathrm{mol} / \mathrm{L}, \mathrm{p}=0.03$; respectively). In addition, several carnitines, C8DC, C16:1, and C18, of the 27 carnitines examined were significantly higher in the HM group than in the control group $(0.07 \pm 0.06$ $\mu \mathrm{mol} / \mathrm{L}$ vs $0.03 \pm 0.01 \mu \mathrm{mol} / \mathrm{L}, \mathrm{p}=0.021 ; 0.18 \pm 0.09 \mu \mathrm{mol} / \mathrm{L}$ vs $0.10 \pm 0.06 \mu \mathrm{mol} / \mathrm{L}, \mathrm{p}=0.03 ;$ and $0.07 \pm 0.02 \mu \mathrm{mol} / \mathrm{L}$ vs $0.03 \pm 0.01 \mu \mathrm{mol} / \mathrm{L}, \mathrm{p}=0.021$; respectively). Conclusion: This study demonstrated that a decrease in some plasma FAAs and an increase in some plasma carnitine levels might be effective in the pathogenesis of HM.
\end{abstract}

Plasma Free Amino Acid and Carnitine Levels in Pregnant Women with Hydatidiform Mole: A Case-Controlled Study

UYANIKOGLU H ${ }^{1}$, KOYUNCU I ${ }^{2}$, SAK $\mathrm{S}^{1}$, INCEBIYIK A ${ }^{1}$, HILALI N ${ }^{1}$, TASDUZEN E ${ }^{1}$, GONEL A ${ }^{2}$, SAK ME ${ }^{1}$.

${ }^{1}$ Harran University Medical Faculty, Department of Obstetrics and Gynecology, Sanliurfa, Turkey

${ }^{2}$ Harran University Medical Faculty, Department of Biochemistry, Sanliurfa, Turkey,

Corresponding author:

Hacer UYANIKOGLU, MD

From the Department of Obstetrics and Gynecology, Faculty of Medicine, Harran University, 63300, Sanliurfa, Turkey

Mail: huoglu@hotmail.com

ORCID ID: http://orcid.org/0000-0003-0316-4900

Tel: +90 5355740425 Fax: +90 4143183192 


\title{
Disclosure of interest
}

The authors report no conflict of interest.

\begin{abstract}
Objective : To compare plasma free amino acid (FAA) and carnitine levels in pregnant women with and without hydatidiform mole (HM).
\end{abstract}

Methods: This prospective study was conducted in patients admitted to Harran University Medical Faculty Hospital Obstetrics Clinic between January and November 2018. Twenty-three pregnant women with HM, and 24 healthy pregnant women as controls were enrolled in the study. The venous blood samples were collected, and, FAA and carnitine concentrations were measured in plasma using liquid chromatography/tandem mass spectrometry (LC-MS/MS).

Results : The levels of alanine, arginine, and valine from the 14 amino acids examined were significantly lower in the HM group than in the healthy group $(475.46 \pm 103.55 \mu \mathrm{mol} / \mathrm{L}$ vs $556.99 \pm 105.16 \mu \mathrm{mol} / \mathrm{L}, \mathrm{p}$ $=0.019 ; 428.87 \pm 138.91 \mu \mathrm{mol} / \mathrm{L}$ vs $547.11 \pm 140.20 \mu \mathrm{mol} / \mathrm{L}, \mathrm{p}=0.009 ;$ and $290.96 \pm 97.10 \mu \mathrm{mol} / \mathrm{L}$ vs $358.61 \pm 75.40 \mu \mathrm{mol} / \mathrm{L}, \mathrm{p}=0.03$; respectively). In addition, several carnitines, C8DC, C16:1, and C18, of the 27 carnitines examined were significantly higher in the HM group than in the control group $(0.07 \pm 0.06$ $\mu \mathrm{mol} / \mathrm{L}$ vs $0.03 \pm 0.01 \mu \mathrm{mol} / \mathrm{L}, \mathrm{p}=0.021 ; 0.18 \pm 0.09 \mu \mathrm{mol} / \mathrm{L}$ vs $0.10 \pm 0.06 \mu \mathrm{mol} / \mathrm{L}, \mathrm{p}=0.03 ;$ and 0.07 $\pm 0.02 \mu \mathrm{mol} / \mathrm{L}$ vs $0.03 \pm 0.01 \mu \mathrm{mol} / \mathrm{L}, \mathrm{p}=0.021 ;$ respectively).

Conclusion : This study demonstrated that a decrease in some plasma FAAs and an increase in some plasma carnitine levels might be effective in the pathogenesis of HM.

Keywords : carnitine, free amino acid, hydatidiform mole, pregnancy, pathogenesis

\section{What is already known about this topic?}

Although there are several studies investigating the pathophysiological mechanism of HM, whether the pathology result from a genetic defects or environmental factors remain controvertial. The pathology of HM is poorly understood.

\section{What does this article add?}

The implication of these findings shed light on understanding the pathogenesis of HM for

further research.

\section{Introduction}

Hydatidiform mole (HM) is a gestational trophoblastic disease, which is characterized by abnormal trophoblastic proliferation and hydrophic degeneration in the placenta [1]. HM may lead to several complications, such as massive bleeding during pregnancy, uterine rupture when performing evacuation, and transforming to gestational trophoblastic neoplasia, so it negatively affects a woman's health both during pregnancy and for rest of her life [2]. Although several studies have identified interesting pathogenetic pathways that might contribute to the development of HM, such as genetic defects in fertilization phase, NLRP7 gene mutations, and dietary changes, the exact ethiology is still not fully elucidated [3-5].

Carnitine (L-3-hydroxy-4-N-N-N-trimethylaminobutyrate) is an essential compound, that is synthesized in only a few organs, such as brain, liver, and kidney. It has a functional role in the intracellular transport of long-chain fatty acids for $\beta$-oxidation process $[6,7]$. Fatty acids are energy substrates that provide the energy necessary for growth, maturation, and transport functions of placenta. Since carnitine biosynthesis in the fetus is insufficient, transport of maternal carnitine through the placenta plays an important role for fetal development [8].

Amino acids are biologically important organic molecules, which play central roles both in building of proteins and as intermediates in metabolism of cells and tissues. The free amino acids (FAAs) are distributed 
throughout the body to participate the active metabolic system [9]. Previous studies have determined that metabolomics analysis, a new method measuring the metabolites in all tissue or biofluid samples, could usable in several diseases which has uncontrolled cell growth [10-12]. In a study in normal pregnant women, the elevated levels of FAAs were found compatible with syncytiotrophoblast activity in the intervillous space [13]. Since it is well known that there are hyperactivity and excess proliferation in trophoblastic cells of molar pregnancies, we hypothesized that FAA levels would be lower in plasma of HM patients than in normal pregnants due to the enhanced metabolism of trophoblastic cells. To the best of our knowledge, this is the first study in the literature which compares plasma FAA and carnitine levels in HM patients with those of healthy pregnant women.

\section{Material and Methods}

This prospective study was conducted between January and November 2018 in the Obstetrics \& Gynecology and Biochemistry Departments of the Harran University Medical Faculty in Sanliurfa, Turkey. This study conformed with the principles of the 2008 Declaration of Helsinki and was approved by the local ethics committee of the Harran University Medical Faculty. Detailed information was provided to all of the women enrolled in this study, and written consent was given by all of the patients.

The study included 23 pregnant women with HM at 7 to 12 weeks of gestation and 24 age and gestational week matched healthy pregnant women. Any individuals with diabetes, thyroid dysfunction or hypertension, multiple gestations, and women whose pathology results were other types of gestational trophoblastic disease, and women who use vitamins or smoke were excluded from the study. The HM diagnosis was made via USG results (Voluson 730 Expert scanner; GE Healthcare, Milwaukee, WI, USA) showing typical complete mole appearance (snowstorm appearance). In addition, patients' ovaries were evaluated in terms of theca lutein cyst during ultrasonographic examination. In all cases, the preoperative diagnosis of HM was confirmed by postoperative histopathology results.

Each woman's age, body mass index (BMI), gravidity, parity, blood pressure, thyroid function test, $\beta$-hcg level, and urine analysis were documented. The venous blood samples were collected preoperatively when the HM diagnosis was made for the biochemical comparisons between the groups.

\section{Blood collection and immunoassay procedures}

Two tubes of blood were collected from all the participants at 7 to 12 weeks of gestation. Blood specimens placed into tubes with anticoagulant were centrifuged at $3000 \mathrm{~g}$ at 4 , for 10 minutes, and, the supernatants were transferred to new tubes and frozen in liquid nitrogen and stored at -80 until analyzed.

\section{Carnitine and Amino Acid Analyses}

Levels of carnitine and FAA in the plasma specimens obtained were measured using LC-MS/MS (Shimadzu 8045, Japan) in line with La Marca et al. and Azzari et al. protocol [14,15].

Briefly, in a new tube, $50 \mu \mathrm{l}$ from each specimen, $50 \mu \mathrm{l}$ internal standard solution, and $700 \mu \mathrm{l}$ Reagent-1 were mixed by vortex for 10 seconds, and the acquired solution was centrifuged at 4,000 rpm for 5 minutes. The data obtained were calculated based on internal standards on Shimadzu Neonatal software. Twenty-seven carnitine and 14 amino acid quantities were analyzed in a single injection.

The following 14 amino acids were analyzed: Methyl Glutaryl, aspartic acid (Asp), glutamic acid (Glu), ornitine (Orn), glycine (Gly), arginine (Arg), argininosuccinic acid (Asa), alanine (Ala), citrulline (Cit), tyrosine (Tyr), valine (Val), methionine (Met), leucine/isoleucine (Leu/lle), and phenylalanine (Phe). The absolute concentration of each amino acid was expressed in $\mu \mathrm{mol} / \mathrm{L}$.

\section{Statistical Analysis}

All analyses were performed using Statistical Packages for Social Sciences for Windows, Version 20.0 (SPSS Inc., NY, USA). The data were expressed as means with standard deviations. Comparisons of the groups for normally distributed variables were performed using an independent samples $t$ test. Differences between 
groups without normal distribution were checked with Mann-Whitney U test, while groups with categorical variables were compared with Pearson chi-square test. Significance was evaluated at $\mathrm{p}$ value $<0.05$ for all tests.

\section{Results}

The demographic and clinical features of the two groups are presented in Table 1. HM patients have more hyperemesis symptoms (nausea and vomiting) than the controls, however, there were no correlation between the urine ketone levels and the serum FAA and carnitine levels in HM group ( $\mathrm{p}>0.05)$. Also, the correlation analysis showed that there were no correlations between the plasma FAAs, carnitine levels, circulating $\beta$-Hcg levels, gestational ages, and maternal ages $(\mathrm{p}>0.05)$.

The outcome of the participants in the control group were followed, and no pregnancy complications were observed during pregnancy. In addition, other parameters releated with birth, such as birth weight and gestational week at delivery, were normal in this group.

Of the 14 amino acids, there were no significant differences in the plasma FAA profiles between the controls and the HM patients, except for alanine, arginine, and valine, their levels were significantly lower in the HM group $(475.46 \pm 103.55$ vs $556.99 \pm 105.16, \mathrm{p}=0.019 ; 428.87 \pm 138.91$ vs $547.11 \pm 140.20, \mathrm{p}=0.009$; and $290.96 \pm 97.10$ vs $358.61 \pm 75.40, \mathrm{p}=0.03$; respectively) (Table 2). In addition, a few of the 27 carnitines examined (i.e., C8DC, C16:1, and C18) were significantly higher in the HM group than in the control group $(0.07 \pm 0.06 \mu \mathrm{mol} / \mathrm{L}$ vs $0.03 \pm 0.01 \mu \mathrm{mol} / \mathrm{L}, \mathrm{p}=0.021 ; 0.18 \pm 0.09 \mu \mathrm{mol} / \mathrm{L}$ vs $0.10 \pm 0.06 \mu \mathrm{mol} / \mathrm{L}, \mathrm{p}=$ 0.03 ; and $0.07 \pm 0.02 \mu \mathrm{mol} / \mathrm{L}$ vs $0.03 \pm 0.01 \mu \mathrm{mol} / \mathrm{L}, \mathrm{p}=0.021$; respectively) (Table 3 ).

The Row-Z score in the heat map system of the FAA levels in maternal plasma of HM patients and healthy pregnants is shown in Fig. 1. The colour change from green to red on the map represents an increase in amino acid levels. In HM patients, 3 of the plasma FAAs (i.e., valine, alanine, and arginine) were found to be lower than those of the controls. The dispersion of carnitine values is demonstrated as a heat map grafic in Fig.2. The red color on the map shows high carnitine values. C8DC, C16:1, and C18 values were significantly higher in the HM group than in the control group.

\section{Discussion}

In this study, we measured plasma FAA and carnitine levels in HM patients and healthy pregnant women as a control group. The original finding of the present study was that three amino acids (i.e., alanine, arginine, and valine) were significantly decreased, and three carnitines (i.e., C8DC, C16:1, and C18) were significantly increased in the pregnant women with HM.

The mean plasma total carnitine concentration in non-pregnant women has been reported to be approximately $40 \mu \mathrm{mol} / \mathrm{L}$ [16]. It has been found that plasma carnitine concentrations decreased during pregnancy and this decrease was mostly in the first half of pregnancy [17]. The reasons for these low plasma carnitine concentrations in pregnancy are currently unknown, but several hypotheses have been asserted: Ringseis et al. speculated that plasma carnitine concentrations may be low in pregnant women due to reduced carnitine synthesis [18]. However, their study had a clear limitation because the number of subjects was very small. Cho and Cha [17] demonstrated that low carnitine levels were caused by increased urinary loss of carnitine in Korean pregnant women. Grube et al. [8] showed that increased expression of carnitine/organic cation transporter 2 (OCTN2), a carnitine-transporting protein in human placenta, can play an important role in carnitine supply of growing fetus especially in early gestation. Grube et al. [8] detected that this protein located in the membrane of syncytiotrophoblast, had a dominant role for carnitine uptake from maternal circulation. However, they have not simultanously measured the carnitine level changes in maternal plasma. Bai et al. [19] have also investigated the reason of carnitine reduction and concluded that OCTN2 mediated L-Car transfer across placenta played a major role in maternal plasma L-Car reduction during pregnancy. We found higher carnitine levels in plasma of pregnant women with HM compared to the healthy pregnant women. This finding suggests a defect in carnitine uptake by syncytiotrophoblasts due to the absence of a healthy pregnancy in HM patients or decreased expression of OCTN2 protein in HM cases. Although we 
did not study OCTN2 protein in pathology specimens of HM patients, this speculation may be a hypothesis for further studies.

Studies on amino acids have been previously published in several cancers and obstetric diseases such as gestational diabetes [20-23]. Neoplastic cells need some amino acids, such as glutamine, glycine, aspartic acid and serine, for the new vessel formation, DNA synthesis, duplication of protein, and synthesis of hormones. In an in vitro study on mice, Roux et al. [12] demonstrated that the glutamine amino acid uptake of pancreatic adenocarcinoma cells increased compared to non-pancreatic tumor cells, therefore, the plasma glutamine levels decreased in the environment. Additionally, Saglik et al. [24] found that glutamine amount was higher in pterygium tissue than in normal conjunctival tissue. Both studies were designed at the tissue level, unlike ours. In our study, circulating plasma glutamine levels were found similar in both groups, however, alanine, arginine, and valine levels were lower in HM group than in healthy pregnants. This discrepancy may be due to different histopathogenesis of the diseases, differences in method used, small sizes of our study, female gender or younger age of our participants.

Liu and colleagues [25] pointed out changes in FAA concentrations in both plasma and gastric fluid, including threonine, serine, alanine, valine, methionine, isoleucine, leucine, tyrosine, phenylalanine, lysine, and arginine in the diagnosis of gastric cancer. They found an increase in gastric fluid FAA levels and a decrease in plasma FAA concentrations in gasric cancer patients compared to nongastric cancer patients. The inconsistency of these metabolic phenotypes between plasma and gastric fluid in gastric cancer patients (opposite FAA levels) was explained as abnormal accumulation of several metabolites in tumor microenvironment. Camelo et al. [13] showed that high FAA levels in the intervillous space were compatible with syncytiotrophoblast activity in healthy pregnant women. They thought that these high levels occurred due to an asymmetric influx or active transport from the trophoblast cells to the blood in the intervillous space. However, there is no study researching the association between the plasma FAAs and HM. As the first study in the literature, we found decreased concentrations in several maternal plasma FAAs in patients with HM. This finding may be caused by impaired active transport from the intervillous space to the blood in patients with HM, or due to excessive accumulation of FAAs in trophoblastic microenvironment, because trophoblastic cells are highly proliferative and are similar to cells in cancer tissue. Although molar pregnancy is not malignant tumor, it has a risk for gestational trophoblastic neoplasia. However, we think that if the tissue-supported studies are performed, it will contribute to our results.

Miyagi et al. [20] suggested that plasma FAA profiling has an important role for cancer screening and diagnosis in patients with asymptomatic early-stage of the disease. Morever, they have demonstrated that the differences in FAA metabolism did not releated with impaired nutritional support of cancer patients. In contrast, some authors have suggested that low plasma FAA levels were associated with anorexia, malnutrition, and progressive weight loss in cancer patients. Thus, the factors affecting plasma FAA levels in these subjects still remain controversial and unclear [10,11]. Our HM patients had impaired nutritional support due to hyperemesis, but there was no correlation between the plasma FAA and urine ketone levels.

Several factors, such as obesity and food intake, may affect the serum FAA and carnitine levels. Rigamonti et al. [26] showed that whey consumption in obese female subjects increased some circulating amino acids (alanine, arginine, asparagine, citrulline, glutamine, hydroxyproline, isoleucine, histidine, leucine, lysine, methionine, ornithine, phenylalanine, proline, serine, threonine, tyrosine and valine). They reported that of these amino acids, isoleucine, leucine, lysine, methionine, phenylalanine, proline, tyrosine, and valine correlatated negatively with starvation and positively with satiety. Additionally, Yamada et al. [27] speculated that deficiencies of certain dietary substrats, such as folic acid, proteins, and vitamin B-12, which are essential for nucleic acid formation in decidual cells, may affect the risk of abortion releated with chromosomal disorders. Although, we did not measure other circulating parameters such as folic acid and vitamins, we showed that HM patients had different plasma FAA and carnitine levels compared to the healthy pregnants. We excluded obese pregnants, smokers, and women who use food supplements from the study to avoid changes in plasma FAA and carnitine levels that may occur due to these factors. However, further epidemiological and biochemical studies are needed to obtain a more precise definition of specific dietary correlation. 
The limitations of this case-control study were that it was designed only between pregnant women and only by measuring the maternal plasma. However, this study is the first in the literature to investigate FAA and carnitine profiling in HM. Although more research is needed to support our results, including larger sample sizes and investigating tissue samples, we think current results may be considered as references for subsequent studies on HM.

\section{Conclusion}

This is the first report showing both plasma FAA and carnitine analyses in HM patients. The results of our study may shed new light on the role of metabolic factors in the pathogenesis of HM.

\section{Acknowledgements}

We thank all the subjects who participated in this study. We also thank Sabuncu T. who is responsible for statistical analysis.

\section{Funding}

No funded.

\section{References}

[1] Landolsi H, Missaoui N, Brahem S, Hmissa S, Gribaa M, Yacoubi MT. The usefulness of p57(KIP2) immunohistochemical staining and genotyping test in the diagnosis of the hydatidiform mole. Pathol Res Pract. 2011;207:498-504.

2 Shamshiri Milani H, Abdollahi M, Torbati S, Asbaghi T, Azargashb E. Risk Factors for Hydatidiform Mole: Is Husband's Job a Major Risk Factor? Asian Pac J Cancer Prev. 2017;18:2657-62.

3 Shih IeM. Gestational trophoblastic neoplasia-pathogenesis and potential therapeutic targets. Lancet Oncol. 2007;8:642-50.

4 Hui P, Buza N, Murphy KM, Ronnett BM. Hydatidiform Moles: Genetic Basis and Precision Diagnosis. Annu Rev Pathol. 2017;12:449-485.

5 Lurain JR. Gestational trophoblastic disease I: epidemiology, pathology, clinical presentation and diagnosis of gestational trophoblastic disease, and management of hydatidiform mole. Am J Obstet Gynecol. 2010;203:531-9.

6 McGarry JD and Brown NF. The mitochondrial carnitine palmitoyltransferase system. From concept to molecular analysis. Eur J Biochem. 1997;244:1-14.

7 Vaz FM and Wanders RJ. Carnitine biosynthesis in mammals. Biochem J. 2002;361:417-29.

8 Grube M, Meyer Zu Schwabedissen H, Draber K, Präger D, Möritz KU, Linnemann K, et al. Expression, localization, and function of the carnitine transporter octn2 (slc22a5) in human placenta. Drug Metab Dispos. 2005;33:31-7.

9 Fiehn O. Combining genomics, metabolome analysis, and biochemical modelling to understand metabolic networks. Comp Funct Genomics. 2001;2:155-68.

10 Lai HS, Lee JC, Lee PH, Wang ST, Chen WJ. Plasma free amino acid profile in cancer patients. Semin Cancer Biol. 2005;15:267-76.

11 Okamoto N, Miyagi Y, Chiba A, Akaike M, Shiozawa M, Imaizumi A, et al. Diagnostic modeling with differences in plasma amino acid profiles between non-cachectic colorectal/breast cancer patients and healthy individuals. Int J Med Med Sci. 2009;1:1-8.

12 Roux C, Riganti C, Borgogno SF, Curto R, Curcio C, Catanzaro V, et al. Endogenous glutamine decrease is associated with pancreatic cancer progression. Oncotarget. 2017;8:95361. 
13 Camelo JS Jr, Jorge SM, Martinez FE. Amino acid composition of parturient plasma, the intervillous space of the placenta and the umbilical vein of term newborn infants. Braz J Med Biol Res. 2004;37:711-7.

14 la Marca G, Malvagia S, Pasquini E, Innocenti M, Fernandez MR, Donati MA, et al. The inclusion of succinylacetone as marker for tyrosinemia type I in expanded newborn screening programs. Rapid Commun Mass Spectrom. 2008;22:812-8.

15 Azzari C, la Marca G, Resti M. Neonatal screening for severe combined immunodeficiency caused by an adenosine deaminase defect: a reliable and inexpensive method using tandem mass spectrometry. J Allergy Clin Immunol. 2011;127:1394-9.

16 Cederblad G, Fahraeus L, Lindgren K. Plasma carnitine and renal carnitine clearance during pregnancy. Am J Clin Nutr. 1986;44:379-83.

17 Cho SW and Cha YS. Pregnancy increases urinary loss of carnitine and reduces plasma carnitine in Korean women. Br J Nutr. 2005;93(5):685-91.

18 Ringseis R, Hanisch N, Seliger G, Eder K. Low availability of carnitine precursors as a possible reason for the diminished plasma carnitine concentrations in pregnant women. BMC Pregnancy Childbirth. 2010;10:178.

19 Bai M, Zeng Q, Chen Y, Chen M, Li P, Ma Z, et al. Maternal plasma L-carnitine reduction during pregnancy is mainly attributed to OCTN2 mediated placental uptake and does not result in maternal hepatic fatty acid $\beta$-oxidation decline. Drug Metab Dispos. 2019;47:582-91.

20 Miyagi Y, Higashiyama M, Gochi A, Akaike M, Ishikawa T, Miura T, et al. Plasma free amino acid profiling of five types of cancer patients and its application for early detection. PLoS One. 2011;6:e24143.

21 Virgiliou C, Gika HG, Witting M, Bletsou AA, Athanasiadis A, Zafrakas M, et al. Amniotic Fluid and Maternal Serum Metabolic Signatures in the Second Trimester Associated with Preterm Delivery. J Proteome Res. 2017;16:898-910.

22 Chorell E, Hall UA, Gustavsson C, Berntorp K, Puhkala J, Luoto R, et al. Pregnancy to postpartum transition of serum metabolites in women with gestational diabetes. Metabolism. 2017;72:27-36.

23 Pappa KI, Vlachos G, Theodora M, Roubelaki M, Angelidou K, Antsaklis A. Intermediate metabolism in association with the amino acid profile during the third trimester of normal pregnancy and diet-controlled gestational diabetes. Am J Obstet Gynecol. 2007;196:65.e1-5.

24 Saglik A, Koyuncu I, Gonel A, Yalcin H, Adibelli FM, Toptan M. Metabolomics analysis in pterygium tissue. Int Ophthalmol. 2019;39:2325-33.

25 Liu J, Lin S, Li Z, Zhou L, Yan X, Xue Y, et al. Free amino acid profiling of gastric juice as a method for discovering potential biomarkers of early gastric cancer. Int J Clin Exp Pathol. 2018;11:2323-36.

26 Rigamonti AE, Leoncini R, De Col A, Tamini S, Cicolini S, Abbruzzese L, et al. The Appetite-Suppressant and GLP-1-Stimulating Effects of Whey Proteins in Obese Subjects are Associated with Increased Circulating Levels of Specific Amino Acids. Nutrients. 2020;12:775.

27 Yamada H, Sata F, Saijo Y, Kishi R, Minakami H. Genetic factors in fetal growth restriction and miscarriage. Semin Thromb Hemost. 2005;31:334-45.

Figure 1 : Heat map analysis showing the distribution of the plasma free amino acid values in both groups. HM: hydatidiform mole, CON: control group. Aspartic acid (Asp), glutamic acid (Glu), ornithine (Orn), glycine (Gly), arginine (Arg), argininosuccinic acid (Asa), alanine (Ala), citrulline (Cit), tyrosine (Tyr), valine (Val), methionine (Met), leucine/isoleucine (Leu/Ile), and phenylalanine (Phe).

Figure 2: Heat map analysis showing the distribution of the plasma carnitine values in both groups. HM: hydatidiform mole, CON: control group. 


\section{Hosted file}

table 1.docx available at https://authorea.com/users/419108/articles/525696-plasmafree-amino-acid-and-carnitine-levels-in-pregnant-women-with-hydatidiform-mole-a-casecontrolled-study

\section{Hosted file}

table 2.docx available at https://authorea.com/users/419108/articles/525696-plasmafree-amino-acid-and-carnitine-levels-in-pregnant-women-with-hydatidiform-mole-a-casecontrolled-study

\section{Hosted file}

table 3.docx available at https://authorea.com/users/419108/articles/525696-plasmafree-amino-acid-and-carnitine-levels-in-pregnant-women-with-hydatidiform-mole-a-casecontrolled-study
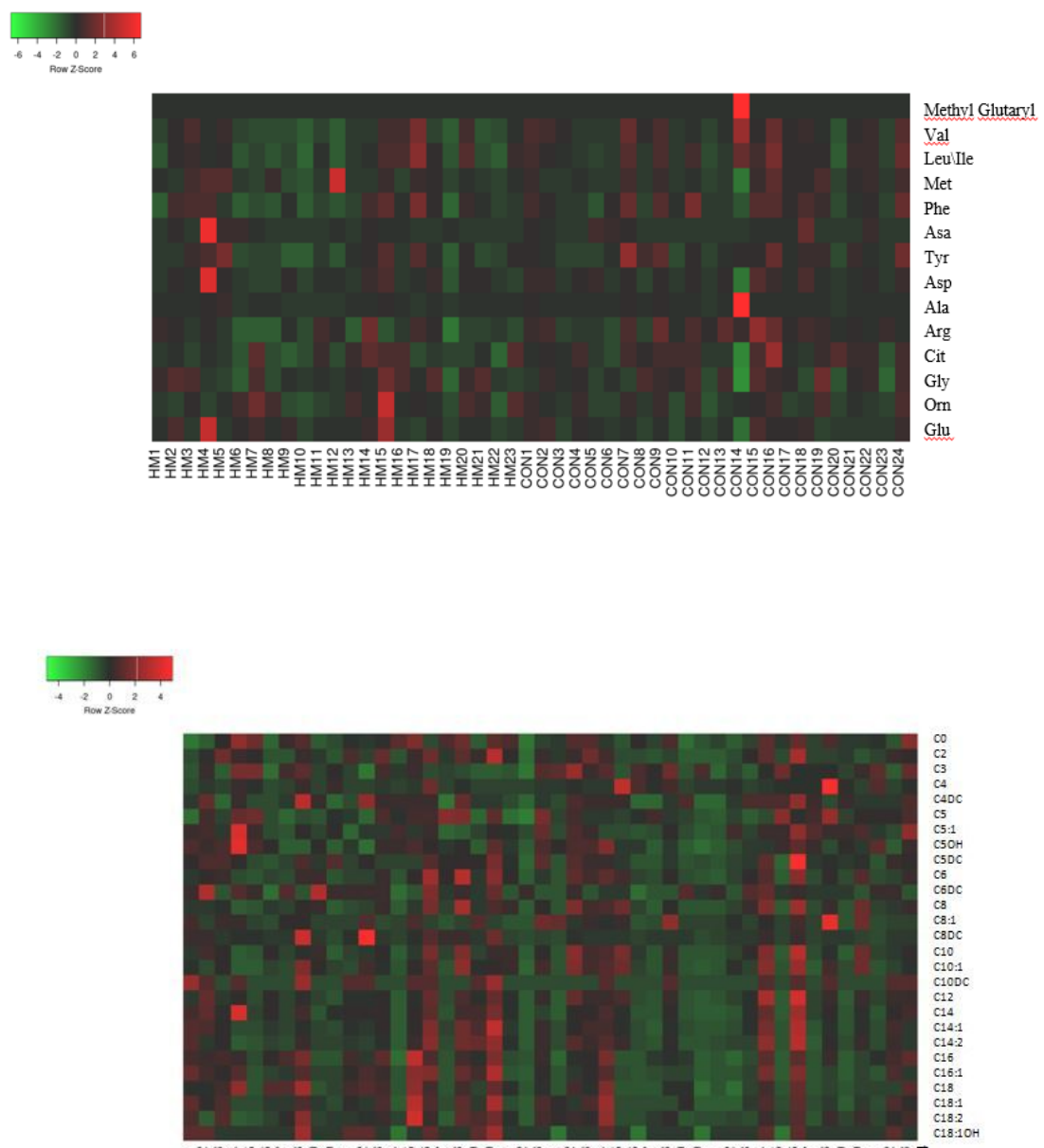

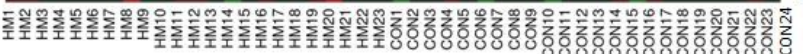

